Working Paper 8403

\title{
FORECASTING USING CONTEMPORANEOUS CORRELATIONS
}

by Michael L. Bagshaw

Working papers of the Federal Reserve

Bank of Cleveland are preliminary materials, circulated to stimulate discussion and critical comment. The views expressed herein are those of the author and not necessarily those of the Federal Reserve Bank of Cleveland or the Board of Governors of the Federal Reserve System.

September 1984

Federal Reserve Bank of Cleveland 
FORECASTING USING CONTEMPORANEOUS CORRELATIONS

Key words: Contemporaneous correlations, forecasting, multivariate time series.

Abstract

In this paper, we present a forecasting technique that uses contemporaneous correlations for forecasting in a time series model when only a subset of the variables are available for the current period. This method potentially provides more accurate forecasts than the standard time series forecasting method, which does not use contemporaneous data. This procedure is illustrated with an example of forecasting the gross national product (GNP), given current $M-1$ in a trivariate autoregressive moving average time series model. Results indicate that during the more stable economic period of 1976: IQ through 1979: IVQ, this method indeed provides forecasts with smaller root mean square errors than the standard forecasts. However, the results during the more turbulent 1980 s are mixed. This latter result indicates that the relationship between the contemporaneous error terms from $\mathbf{M}-1$ and GNP changed during this period. However, the results for the period 1983:IIQ through 1984:IIQ indicate that the relationship may have returned to pre-1980 form. The forecast errors during this latter period had smaller root mean square errors when the contemporaneous errors were used. 


\section{Introduction}

When forecasting in a multivariate framework, there are occasions when data are available only for a subset of the variables in the model for the current time period. It is desirable to use the additional information in this subset of known variables to forecast values that are not currently available. This includes both values of the unknown variables in the current period and future values of all variables. In multiple time series models, any contemporaneous correlations among the variables are modeled as part of the error structure. Consequently, the standard forecasts generated by these models cannot use the information from current data in developing forecasts. However, the information in the contemporaneously correlated error terms can be used to obtain forecasts with smaller error variances. This paper explains how these forecasts can be obtained and presents an example where the contemporaneous correlation between the money supply (M-1) and nominal GN is used to reduce the root mean square error (RMSE) in forecasting current and future GNP, given current M-1. While this paper focuses on the multivariate APMA time series models, the results hold for any multivariate models that do not explicitly model the contemporaneous correlations.

\section{Multivariate ARMA Time Series Models}

The following is a very brief description of multivariate autoregressive moving average time series models (ARMA); Tiao and Box (1981) provide a more detailed description. The ARMA models can include seasonal 
components; however, this paper uses only examples of nonseasonal models and, thus, presents only a description of the nonseasonal models. The general nonseasonal multivariate ARMA model of order $(p, q)$ is given by

(1) $\underline{\phi}_{p}(B) \underline{z}_{t}=\underline{\theta}_{q}(B) \underline{a}_{t}+\underline{\theta}_{0}$,

where

(2)

$$
\begin{aligned}
& \underline{\phi}_{p}(B)=I-\phi_{1} B-\ldots-\underline{\phi}_{p} B^{p} \\
& \ddot{\theta}_{q}(B)=I-e_{-}^{e} B-\ldots-\theta_{-4} B^{q},
\end{aligned}
$$

where

$$
\begin{aligned}
& \left.B=\text { backshift operator ( } i . e ., B^{S} z_{i, t}=z_{i, t-s}\right), \\
& \underline{I}=k \times k \text { identity matrix, } \\
& \underline{z}=\text { vector of } k \text { variables in the model, } \\
& \underline{\phi}_{j}{ }^{\prime} s \text { and } \underline{\theta}_{j}{ }^{\prime} s=k \times k \text { matrixes of unknown parameters, } \\
& \underline{e}_{0}=k \times 1 \text { vector of unknown parameters, and } \\
& \underline{a}=k \times 1 \text { vector of random errors that are identically and } \\
& \quad \text { independently distributed as } N(0, \Sigma) .
\end{aligned}
$$

Thus, it is assumed that the $a_{j, t}$ 's at different points in time are independent but not necessarily that the elements of $\underline{a}_{t}$ are independent at a given point in time.

The $n$-period-ahead forecasts from these models at time $\mathbf{t}\left(\underline{z}_{t}(n)\right)$ are given by 
(3)

$$
\begin{aligned}
\underline{z}_{t}(n)=\underline{\phi}_{1}\left[\underline{z}_{t+n-1}\right] & +\ldots+\underline{\phi}_{p}\left[\underline{z}_{t+n-p}\right] \\
& +\left[\underline{a}_{t+n}\right]-\underline{\theta}_{1}\left[\underline{a}_{t+n-1}\right]-\ldots-\underline{\theta}_{q}\left[\underline{a}_{t+n-q}\right],
\end{aligned}
$$

where for any value of $t, n, m,\left[\underline{x}_{t+n-m}\right]$ implies the conditional expected values of the random variables $\underline{x}_{t+n-m}$ at time $t$. If $n-m$ is less than or equal to 0 , then the conditional expected values are the actual values of the random variables and the error terms. If $n-m$ is greater than 0 , then the expected values are the best forecasts available for these random variables and error terms at time t. Because the error terms are uncorrelated with present and past information, the best forecasts (in standard time series forecasting) of the error terms for $n-m$ greater than 0 are their conditional means, which are 0 . The forecasts can be generated iteratively with the one-period-ahead forecasts depending only on known values of the variables and error terms. The longer-length forecasts in turn depend on the shorter-length forecasts.

\section{Using Contemporaneous Correlations}

If some of the variables at time $\mathbf{t}+\mathbf{I}$ are known, then the expected values of the associated error terms are not necessarily 0 if there is contemporaneous correlation between the error terms. (In the following, the term current stands for time $t+1$, $\mathbf{i} . e .$, the time period for which the final value of some of the variables is known but not all.) For example, consider the following simple model:

$$
\begin{aligned}
& z_{1, t}=\phi_{21} z_{2, t-1}+a_{1, t}, \\
& z_{2, t}=\phi_{12} z_{1, t-1}+a_{2, t},
\end{aligned}
$$


where each error term has a variance of 1 and the contemporaneous correlation between $a_{1}$ and $a_{2}$ is 0.5 . The standard $n$-period-ahead forecasts at time $\mathbf{t}$ from this model are given by

$$
\begin{aligned}
& z_{1, t}(n)=\phi_{21}\left[z_{2, t+n-1}\right]+\left[a_{1, t+n}\right] \\
& z_{2, t}(n)=\phi_{12}\left[z_{1, t+n-1}\right]+\left[a_{2, t+n}\right]
\end{aligned}
$$

where the conditional expected values of the error terms are 0 in the standard multivariate forecasts.

In particular, the one-period-ahead forecasts are

$$
\begin{aligned}
& z_{1, t}(1)=\phi_{21} z_{2, t}+\left[a_{1, t+1}\right], \\
& z_{2, t}(1)=\phi_{12} z_{1, t}+\left[a_{2, t+1}\right] .
\end{aligned}
$$

Thus, in standard time series forecasting, the one-period-ahead forecast of $z_{2}, t+1$ is $\phi_{12} z_{1, t}$, which has a standard error of $\sigma_{2}$ (the standard error of $a_{2}$ ), which for this model is assumed to be 1 . However, if $z_{1, t+1}$ is known and $z_{2}, t+1$ is not, then a better forecast of $z_{2, t+1}$ can be obtained by using the contemporaneous correlation between $a_{1}$ and $a_{2}$. This is true because $a_{1, t+1}$, which equals the forecast error made at time $t$ for $z_{1}$ at time $t+1\left(a_{1, t+1}=z_{1, t+1}-z_{1, t}(1)\right)$, is known. Thus, the correlation between $a_{1}$ and $a_{2}$ can be used to estimate $a_{2, t+1}$ given $a_{1, t+1}$. For this model, the relationship (derived from the variance-covariance matrix of the error terms) between the two error terms is given by 
(4) $a_{2, t}=0.5 * a_{1, t}+e_{2, t}$,

where $e_{2, t}$ is the error in predicting $a_{2, t}$ using $a_{1, t}$. For this model, this error will have a variance of 0.75 . Thus, given $z_{1, t+1}$, the best forecast of $a_{2, t+1}$ is given by $0.5{ }^{*} a_{1, t+1}$; consequently, the forecast for $z_{2, t+1}$ is modified to be

(5)

$$
z_{2, t}(1)=\phi_{12} z_{1, t}+0.5 * a_{1, t+1}
$$

This forecast will have an error of $e_{2, t+1}$, which has a variance of 0.75 compared with the error variance of 1 for the forecast from the original model, using only $z_{1, t}$ to forecast $z_{2, t}$. Thus, knowing $z_{1, t+1}$ reduces the error variance in forecasting $z_{2, t+1}$ by 25 percent for this example. Also, the forecast using contemporaneous data will remain unbiased. In this model, because the forecast of $\mathbf{z}_{1}$ depends on $z_{2}$, this reduction in forecast error variance for one-period-ahead forecasts of $z_{2}$ will lead to a reduction of forecast error variance for forecasts of $z_{1}$. For example, the one-period-ahead forecast at time $t+1$ for $z_{1}$ using contemporaneous correlations is given by

$$
\begin{aligned}
z_{1, t+1}(1) & =\phi_{21}\left[z_{2, t+1}\right]+\left[a_{1, t+2}\right] \\
& =\phi_{21} *\left(\phi_{12} z_{1, t}+0.5 * a_{1, t+1}\right) .
\end{aligned}
$$


This forecast has an error $e_{1, t+2}$ given by

$$
\begin{aligned}
e_{1, t+2} & =z_{1, t+2}-z_{1, t+1}(1) \\
& =\phi 21 * z_{2, t+1}+a_{1, t+2}-\left(\phi_{21} *\left(\phi_{12} z_{1, t}+0.5^{*} a_{1, t+1}\right)\right. \\
& =\phi_{21}{ }^{*}\left(z_{2, t+1}-\left(\phi_{12} z_{1, t}+0.5^{*} a_{1, t+1}\right)+a_{1, t+2}\right. \\
& =\phi_{21}{ }^{*} e_{2, t+1}+a_{1, t+2} .
\end{aligned}
$$

Similarly, the forecast error $a_{1, t+1}(1)$ for $z_{1, t+2}$ without using contemporaneous data is

$$
a_{1, t+1}(1)=\phi_{21} * a_{2, t+1}+a_{1, t+2}
$$

Thus, the one-period-ahead forecast of $z_{1}$ using contemporaneous data has a variance of $\sigma_{1}^{2}+\phi_{21}^{2} *_{\sigma_{2}}^{2}=1+0.75 * \phi_{21}^{2}$ while the forecast variance not using contemporaneous data has a variance of $\sigma_{1}^{2}+\phi_{21}^{2} *_{\sigma_{2}}^{2}=1+\phi_{21}^{2}$. Similarly, it can be shown that there is a reduction in the forecast variance for future periods when using contemporaneous correlations for this model. Thus, the use of contemporaneous data reduces not only the forecast error of the contemporaneous value of the other variable in this model but also the forecasts of the observed variable in future periods.

In general, the amount of reduction in the contemporaneous period and future periods will depend on the correlations between the error terms in the known variables and those in the unknown variables, as well as the structure of the model. 
In the general case, the variables can be divided into two sets -those for which information is available for the current time period (LA) and those for which current information is not available $\left(\underline{z}_{N A}\right)$. In the following discussion, it is assumed that the first $k_{A}$ variables have data available for the current time period and that the remaining $k-k_{A}$ variables do not. The errors in the second group then can be forecast from the known errors in the first group. The relationship between the unknown errors and the known errors $\left(\underline{a}_{A, t}-a k_{A} \times 1\right.$ vector) can be represented as

(6) $a_{i, t}=\underline{b}_{i-A} a_{A} t+e_{i, t} \quad$ for $i=k_{A^{+}} 1, k_{A^{+}}, \ldots, k$,

where $\underline{h}_{j}$ will be a 1 x k vector of estimated coefficients. Because the error terms in equation 6 generally will be correlated across equations (that is, $e_{\mathbf{i}, t}$ and $e_{j, t} w i l l$ generally be correlated), these estimates should be determined by using a generalized least squares estimator (Theil 1971).

Given the set of equations presented in equation 6, the forecasts (or, equivalently, the conditional expected values) of the unknown errors $a_{i, t+1}$ are

(7) $a_{i, t}(1)=\underline{b}_{i} \underline{a}_{A, t+1}$ for $\mathbf{i}=k_{A}+1, k_{A}+2, \ldots, k$.

The forecasts using contemporaneous correlations are thus given by equation 3, where $\left[\underline{a}_{A}, t+n-j, \underline{a}_{N A}, t+n-j\right]=(\underline{1})$ if $n-j$ is greater than 1, $\left(\underline{a}_{A, t+1}, \underline{b}_{i} \underline{a}_{A}, t+1\right)$ if $n-j=1$ (versus a forecast of 1 for the unknown variables i n normal multivariate time series forecasting), and $\underline{a}_{t+n-j}$ if $n-j$ 
is less than 1. Thus, the basic change in the forecasts is that the conditional expected value of $a_{j, t+1}\left(i=k_{A+1}, k_{A+2}, \ldots, k\right)$ is estimated from the known errors $\left(a_{A}, t+1\right)$. This has an obvious impact on the forecasts at time $t+1$. However, as previously illustrated, it also may improve the forecasts for longer time periods for all variables, because future forecasts for all variables may depend on the forecasts at time $t+1$.

\section{Forecasting GN Using Contemporaneous $M-1$}

To illustrate this method of forecasting, we have applied it to a three-variable, quarterly model that was estimated in another ongoing research project (Bagshaw and Gavin 1984). The variables in this model are the money supply M-1, GP in current dollars, and the bond-equivalent yield on Treasury bills with three months to maturity (RTB3). M-1 and GNP are both seasonally adjusted and measured in billions of current dollars; RTB3 is not seasonally adjusted and is measured in percentage points. The model was estimated in $\ln (1+$ RTB3 $)$ and in changes in the natural logarithm of $M-1$ and GNP, or $\nabla \ln (M-1)$ and $\nabla \ln ($ GNP)). Because of the credit controls during 1980, the shift in monetary policy during the 1980s, and the Depository Institutions Deregulation and Monetary Control Act of 1980, there is some question whether the last three or four years would be adequately represented by a model estimated over an earlier time period. Consequently, we developed time series models covering two overlapping time periods to test for this problem. The two time periods were from 1959: IQ through 1976:IVQ and 1959: IQ through 1979:IVQ. These allow forecasts to be produced for the time periods from 
1977: IQ through 1979:IVQ and 1980: IQ through 1984:IIQ. If these changes did indeed have an impact, then the earlier period should be more stable than the latter period and the model should perform better during this period relative to the latter period.

The models were estimated using the Tiao-Box procedure to estimate the parameters of a multivariate simultaneous equation model. The procedure is an interactive one similar in principle to that used in single Box-Jenkins modeling (Box and Jenkins 1976). The steps involved are (1) tentatively identify a model by examining autocorrelations and cross-correlations of the series; (2) estimate the parameters of this model; and (3) apply diagnostic checks to the residuals. These diagnostic checks include checks of correlations in the residuals, normality of residuals, etc. If the residuals do not pass the diagnostic checks, then the tentative model is modified and steps 2 and 3 are repeated. This process continues until a satisfactory model is obtained.

When applied to the two time periods, this technique resulted in the same functional form for the model, with slightly different estimated parameters. The resulting model was

$$
\begin{aligned}
\nabla \ln \left(M-1_{t}\right) & =\phi_{11}{ }^{\star} \nabla \ln \left(M-1_{t-1}\right)-\theta_{12}{ }^{\star} a_{2, t-1}+a_{1, t}, \\
\ln \left(1+R T B 3_{t}\right) & =\phi_{22}{ }^{\star} \ln \left(1+R T B 3_{t-1}\right)+a_{2, t}-\theta_{22}{ }^{\star} a_{2, t-1}, \\
\nabla \ln \left(G P_{t}\right) & =\phi_{31}{ }^{\star} \nabla \ln \left(M-1_{t-1}\right)-\theta_{31}{ }^{\star} a_{3, t-1}+a_{3, t} .
\end{aligned}
$$

All of the estimated parameters of the model are significant at the 0.001 level (see table 1). There are no significant correlations remaining in the 
residuals, except for the contemporaneous correlation between the error terms in M-1 and GNP, and the models passed the usual diagnostic tests.

Because only the contemporaneous correlation between the error terms in GNP and $M-1$ was significant, we developed a forecast of the error in the GN equation given only the error in the M-1 equation. That is, even though the data for RTB3 were available even before the data for $M-1$, these data do not provide useful information in forecasting either contemporaneous GP or M-1. (In fact, we estimated regressions using both contemporaneous errors in M-1 and RTB3 as independent variables and the error in GNP as the dependent variable. The RTB3 term was not significant at the 0.05 percent level, and the addition of the RTB3 term did not significantly improve the regression.) The resulting relationship was

$$
a_{3, t}=b_{1} \star a_{1, t}+e_{1, t}
$$

where $\mathrm{b}_{1}$ was estimated to be 0.792 in the period through 1976: IVQ and 0.768 in the period through 1979:IVQ. The standard deviation of $e_{1}$ was 0.0078 versus a standard deviation of 0.0089 for $\mathrm{a}_{3}$ in the period through 1976:IVQ and 0.0080 versus 0.0090 in the period through 1979:IVQ. Thus, we would expect approximately a 12 percent reduction in the RMSE from forecasting GNP when contemporaneous $M-1$ is available, compared with using only lagged $M-1$.

To test the results on out-of-sample forecasts, we used the model fitted through 1976: IVQ to forecast GP over the period 1977:IQ through 1979:IVQ. In one case, we used only lagged $M-1$ and in the second case, we used contemporaneous M-1. Also, we used the model fitted through 1979: IVQ to 
forecast over the period 1980: IQ through 1984: IIQ. In all cases, the models were used to forecast both contemporaneous changes in $\ln ($ GNP) and changes in $\ln$ (GNP) from time $t$ to time $t+2$, that is, the change from the last known value of $\ln (G N P)$ to the forecast value for two quarters after that known value occurred (see table 2).

From these results, we see that during the period 1977: IQ through 1979: IVQ there was a decrease in RMSE of roughly 8 percent using contemporaneous M-1 in forecasting current GNP, as compared with using only lagged $M-1$. This agrees quite well with the theoretical reduction of 12 percent. However, during the period 1980: IQ through 1984: IIQ the RMSE was actually marginally larger using contemporaneous $\mathrm{M}-1$. For the two-quarter change forecasts, there was a reduction of roughly 4 percent in the first period and an increase of roughly 3 percent in the latter period.

As mentioned earlier in this paper, the 1980s is a time when many events would potentially affect the relationships among $M-1$, GNP, and RTB3. However, it is thought that these events have changed again from roughly 1983: IIIQ to the present in such a way that the relationships would be back to pre-1980 form. Table 3 presents the contemporaneous forecast errors from 1980:IQ to 1984:IIQ. From these errors, we see that during the period of 1983: I IQ through 1984: IIQ the forecast using contemporaneous $M-1$ has done much better than the one using only lagged M-1. In fact, the RMSE for the forecasts using only lagged $M-1$ was 0.0131 over this period, while that for the forecasts using contemporaneous $M-1$ was 0.0101 , a reduction of approximately 22 percent. For the two-quarter change forecasts, the time period over which the latest change would affect the outcome is from 1983:IVQ 
to 1984:IIQ, because the two-quarter change for 1983:IIIQ depends on the data from 1983:IIQ. The RMSE for the period 1983:IIQ through 1984:IIQ using only lagged $M-1$ was 0.0210 ; that for the forecast using contemporaneous $M-1$ was 0.0188. Thus, using contemporaneous $M-1$ reduced the RMSE for this period by roughly 10 percent.

\section{Summary}

Because multivariate time series models include the contemporaneous correlations in the error structure, these models do not use contemporaneous data to forecast variables that are not available for the current period. However, as demonstrated in this paper, these data can successfully be used to obtain more accurate forecasts for the current and future time periods. Indeed, forecasts of GNP using contemporaneous M-1 had roughly an 8 percent smaller RMSE during the period 1977:IQ through 1979: IVQ than that using only lagged M-1. The results during the period 1980: IQ through 1984:IIQ are mixed. However, the results indicate that using contemporaneous M-1 provided more accurate forecasts during 1983:IIQ through 1984:IIQ, suggesting that using contemporaneous $M-1$ data would provide more accurate forecasts in the future. 
Table 1 Estimated Parameters

Time period

\begin{tabular}{ccc} 
Parameter & $1959:$ IQ $-1976:$ IVQ & $1959:$ IQ $-1979:$ IVQ \\
\hline$\phi_{11}$ & 0.9280 & 0.9230 \\
$\phi_{22}$ & 1.0020 & 1.0300 \\
$\phi_{31}$ & 1.5540 & 1.5530 \\
$\theta_{12}$ & 1.2490 & 1.2530 \\
${ }_{22}$ & -0.4650 & -0.5140 \\
${ }_{31}$ & 0.9190 & 0.8990
\end{tabular}

\section{Standard}

deviations and correlations
$\sigma_{1}$
0.0054
0.0054
$\sigma_{2}$
0.0013
0.0014
$\sigma_{3}$
0.0089
0.0090
${ }^{\rho} 12$
$-0.0300$
$-0.1300$
${ }^{\circ} 13$
0.4600
0.4500
$\rho_{23}$
$-0.1600$
$-0.2100$ 
Table 2 Forecast Error Statistics for GNP using M-1

Time period

\begin{tabular}{|c|c|c|c|c|}
\hline & \multicolumn{2}{|c|}{ 1977:IQ - 1979:IVQ } & \multicolumn{2}{|c|}{$1980: I Q-1984: I I Q$} \\
\hline \multicolumn{5}{|c|}{ Contemporaneous forecasts } \\
\hline & Lagged & Contemporaneous & Lagged & Contemporaneous \\
\hline Mean error & 0.0009 & -0.0003 & -0.0072 & -0.0080 \\
\hline RMSE & 0.0095 & 0.0087 & 0.0142 & 0.0143 \\
\hline $\mathrm{MAE}$ & 0.0068 & 0.0068 & 0.0122 & 0.0129 \\
\hline
\end{tabular}

Two-quarter change forecasts

\begin{tabular}{|c|c|c|c|c|}
\hline & Lagged & Contemporaneous & Lagged & Contemporaneous \\
\hline Mean error & 0.0036 & 0.0010 & -0.0155 & -0.0157 \\
\hline RMSE & 0.0114 & 0.0109 & 0.0260 & 0.0267 \\
\hline MAE & 0.0093 & 0.0093 & 0.0212 & 0.0214 \\
\hline
\end{tabular}

NOTE: RMSE - root mean square error of the forecast.

MAE - mean absolute error of the forecast. 
Table 3 Forecast Errors for GNP using M-1 for 1980: IQ through 1982: IVQ

\begin{tabular}{|c|c|c|c|c|}
\hline \multirow{2}{*}{$\begin{array}{l}\text { Time } \\
\text { period }\end{array}$} & \multicolumn{2}{|c|}{$\begin{array}{c}\text { Contemporaneous } \\
\text { forecast }\end{array}$} & \multicolumn{2}{|c|}{$\begin{array}{l}\text { Two-quarter change } \\
\text { forecast }\end{array}$} \\
\hline & Lagged & Contemporaneous & Lagged & Contemporaneous \\
\hline 1980:IQ & 0.0007 & -0.0102 & -0.0167 & -0.0066 \\
\hline 1980: IIQ & -0.0125 & 0.0080 & -0.0118 & -0.0227 \\
\hline 1980: I I IQ & 0.0163 & -0.0115 & 0.0038 & 0.0242 \\
\hline 1980: IVQ & 0.0080 & 0.0125 & 0.0243 & -0.0035 \\
\hline $1981: I Q$ & 0.0045 & 0.0060 & 0.0126 & 0.0170 \\
\hline 1981: IIQ & -0.0049 & -0.0086 & -0.0003 & 0.0012 \\
\hline 1981: I I I & 0.0046 & 0.0107 & -0.0003 & -0.0040 \\
\hline 1981: IVQ & -0.0130 & -0.0146 & -0.0084 & -0.0022 \\
\hline 1982:IQ & -0.0165 & -0.0200 & -0.0295 & -0.0311 \\
\hline 1982:IIQ & -0.0237 & -0.0156 & -0.0402 & -0.0437 \\
\hline 1982: I IIQ & -0.0117 & -0.0148 & -0.0354 & -0.0273 \\
\hline $1982:$ I VQ & -0.0105 & -0.0229 & -0.0222 & -0.0253 \\
\hline 1983: IQ & -0.0235 & -0.0198 & -0.0340 & -0.0464 \\
\hline 1983: I I & -0.0241 & -0.0243 & -0.0476 & -0.0439 \\
\hline 1983: I I IQ & -0.0202 & -0.0176 & -0.0443 & -0.0445 \\
\hline 1983: IVQ & -0.0140 & -0.0079 & -0.0343 & -0.0318 \\
\hline 1984 : IQ & 0.0090 & -0.0059 & -0.0050 & 0.0011 \\
\hline 1984 : I IQ & 0.0018 & 0.0016 & 0.0108 & 0.0077 \\
\hline
\end{tabular}


$\underline{\text { References }}$

Bagshaw, Michael L., and William T. Gavin. "Velocity: A Multivariate Time Series Approach," Working Paper. Federal Reserve Bank of Cleveland, forthcoming.

Box, G.E.P., and G.M. Jenkins. Time Series Analysis, Forecasting and Control, San Francisco: Hol den-Day, 1976.

Theil, Henri. Principles of Econometrics, Now York: John Wiley \& Sons, Inc., 1971.

Tiao, G.C., and G.E.P. Box. "Modeling Multiple Time Series with Applications," Journal of the American Statistical Association, vol. 76, no. 376 (1981), pp. 802-16. 